\title{
cAMP Responsive Element-like Sequences Are Detected in the Upstream Region of a Mating Gene of the Green Alga, Chlamydomonas reinhardtii
}

\author{
Hidenobu Uchida ${ }^{1, *}$, Kensaku Suzuki ${ }^{1}$, Goro Tanifuji ${ }^{2}$, Tomoya Yamaguchi ${ }^{1}$, \\ Osami Misumi ${ }^{3}$, Tsuneyoshi Kuroiwa ${ }^{3}$ and Yoshiaki Hara ${ }^{2}$ \\ ${ }^{1}$ Plant Physiology Laboratory, National Agricultural Research Center for Tohoku Region, Morioka 020-0198, Japan \\ ${ }^{2}$ Department of Biology, Faculty of Science, Yamagata University, Yamagata 990-8560, Japan \\ ${ }^{3}$ Department of Life Science, College of Science, Rikkyo University, Tokyo 171-8501, Japan
}

Received September 29, 2003; December 30, 2003

\begin{abstract}
Summary It is well known that after mixing gametes of two mating types of the green alga Chlamydomonas reinhardtii, intracellular cAMP concentration increases within $1 \mathrm{~min}$, and zygotes are formed within $10 \mathrm{~min}$. We have shown that within $10 \mathrm{~min}$ after the mixing, zys genes are expressed. In the present study, we isolated and determined the sequence for a zys 3 genomic clone. Comparing this $5^{\prime}$ upstream region with those of $z y s 1 B$ and $z y s 4$, we detected putative AP-1-related elements including cyclic AMP responsive elements in three upstream gene regions.
\end{abstract}

In mating of heterothallic Chlamydomonas reinhardtii, zygotes are formed synchronously from the large numbers of gametes, more than $85 \%$ of $10^{8}$ to $10^{9}$ gametes fusing to form quadriflagellates (zygotes). Within $20 \mathrm{sec}$ after mixing of Chlamydomonas gametes, intracellular cAMP concentration increases (Pijst et al. 1984, Pasquale and Goodenough 1987). Recently, a kinase, which is translocated to the flagella of gametes, was isolated (Pan and Snell 2000). These findings suggested the presence of putative signaling pathways induced upon mating. Though mating-induced genes, expressed within 10 min after the mixing, have been reported (Ferris and Goodenough 1987, Uchida et al. 1993), there have been no reports on cAMP related upstream elements in mating genes in green algae, or higher plants.

We have been interested in morphological changes and genes expressed within 10 min after the initiation of mating in Chlamydomonas zygote formation. In fact, we have identified several genes by cDNA cloning (Uchida et al. 1993). One of these genes, zys3, was alternatively transcribed and encodes proteins with ankylin repeats involved in protein-protein interactions (Kuriyama et al. 1999). In order to better understand the gene structure and putative motifs upstream of the zys 3 gene, we have isolated and sequenced a genomic clone, and searched the $5^{\prime}$ upstream nucleotide sequence for transcription factor binding sites related to cAMP response in this study.

\section{Materials and methods}

Nucleotide sequences of subclones were determined with an automated DNA-sequencing systems 310 and 373 (Applied Biosystems) using Dye Terminator Cycle Sequencing Kit or Dye Primer Cycle Sequencing Kit (Applied Biosystems, Foster City, CA, USA). Sequences were analyzed with DNASIS software (Hitachi Software Engineering, Yokohama, Japan). The zys 3 genomic sequence was deposited in DDBJ, EMBL, GenBank with an Accession number of AB091098. In order to detect transcription element binding sites, nucleotide sequences were analyzed with searches in TESS (Transcription Element Search Software- on the WWW, Jonathan Schug and G. Christ-

\footnotetext{
* Corresponding author, e-mai: huchida@ishikawa-c.ac.jp
} 
ian Overton, Technical Report CBIL-TR-1997-1001-v0.0, of the Computational Biology and Informatics Laboratory, School of Medicine, University of Pennsylvania, 1997).

\section{Results and discussion}

In order to isolate a genomic clone of zys3 gene, a Chlamydomonas reinhardtii genomic library (Uchida et al. 1999) was screened using a 1.3-kb cDNA insert of pZS1860 (Uchida et al. 1993) as a probe. Approximately $1.1 \times 10^{4}$ recombinant clones were screened. Southern blotting analysis, restriction mapping and partial nucleotide sequencing showed that a subclone of phage clone pC3-4 included the zys3 gene (Uchida et al. 2001).

A region of 5-kb nucleotides was sequenced which included the zys 3 gene in two subclones pC34Eco4.8 and pC34Sal5.6 (Fig. 1). The sequence determined in this study included 1477-bp of $5^{\prime}$ upstream and 174-bp of $3^{\prime}$ downstream regions flanking $3405 \mathrm{bp}$ of the region corresponding to a full-length cDNA clone, T106 (GenBank accession number AB004042). The zys 3 gene possessed 5 introns, with length of 438, 229, 535, 413 and $245 \mathrm{bp}$. Ankyrin repeats (amino acids 33-150) was located over exons 1, 2 and 3. Introns 2, 3, 4 and 5 included G- and C-biased spans adjacent to the $5^{\prime}$ and $3^{\prime}$ boundaries, respectively, while this tendency was not observed in intron 1 (Fig. 2). During the present study, another research group submitted a zys 3 partial genomic sequence lacking the $3^{\prime}$ end of the exon (GenBank accession number AF385612, version AF385612.1 GI:14550125). Comparing this sequence to our sequence, nine one-base insertions at base positions 753, 796, 1238, $1418,1425,1747,1999,2001$ and 3018 at the $5^{\prime}$ end (NcoI site) of the 5-kb sequence, eight substitutions at 907, 3064, 3089, 3240, 3241, 3672, 3788 and 3794, and two one-base deletions at the positions 799-800 and 1828-1829 were found. The nucleotide differences in this gene may be due to the strain difference between $137 \mathrm{c}$ used in our study and the strain used by the previous research group, CC620/CC621 (Woessner and Goodenough 1989).

In Chlamydomonas, physiological and subsequent morphological phenomena are commonly observed during fertilization (Gilbert 1988, Harris 1989). After mixing two types of Chlamydomonas reinhardtii gametes, intracellular cAMP increased within a minute, and expression of zys genes starts within ten minutes. In mammals, cAMP responsive elements (CREs) are located in upstream regions of genes whose expression is regulated by a cAMP signal. In order to check possible detection of CREs in zys3, the 1477-bp of the $5^{\prime}$ upstream region was analyzed with searches in TESS. In two positions of the zys 3 upstream region, we detected putative CRE-related motifs with log-likelihood scores of more than 12.0, and these scores were above the secondary threshold;

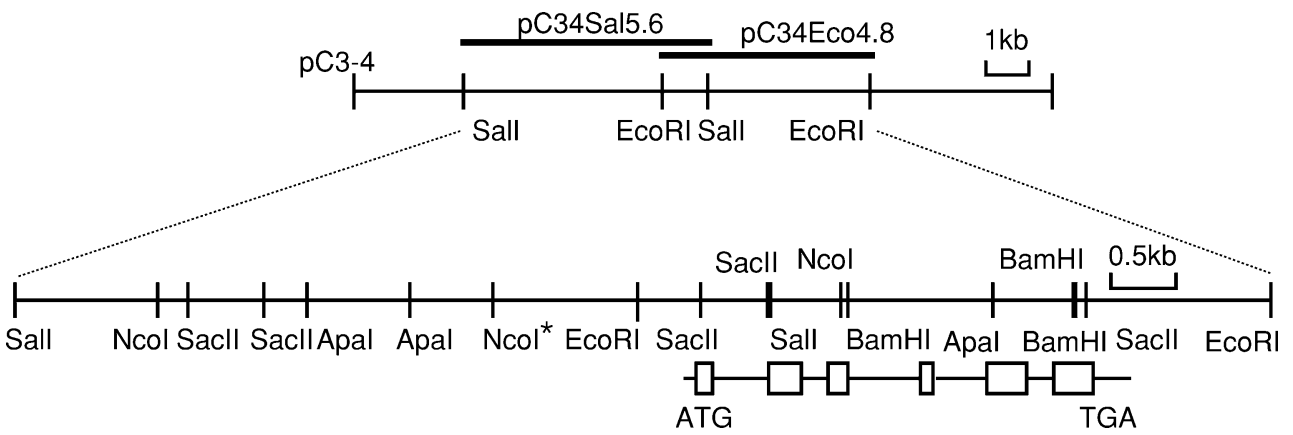

Fig. 1. Restriction maps of the $16-\mathrm{kb}$ genomic clone showing the location and organization of the zys 3 gene. The upper horizontal line with vertical lines shows the restriction map of the 16-kb genomic clone of phage clone pC3-4, whereas lower one indicates an expanded view of the 9.4-kb SalI/EcoRI fragment. Locations of subclones referred in the text are shown at the top. Positions of exons, start and stop codons are shown at the bottom, with open boxes, ATG, and TGA, respectively. The NcoI site at the $5^{\prime}$ end of the zys 3 sequence is shown with an asterisk. Map scale bars are shown at the right of the panel. 
Intron 1

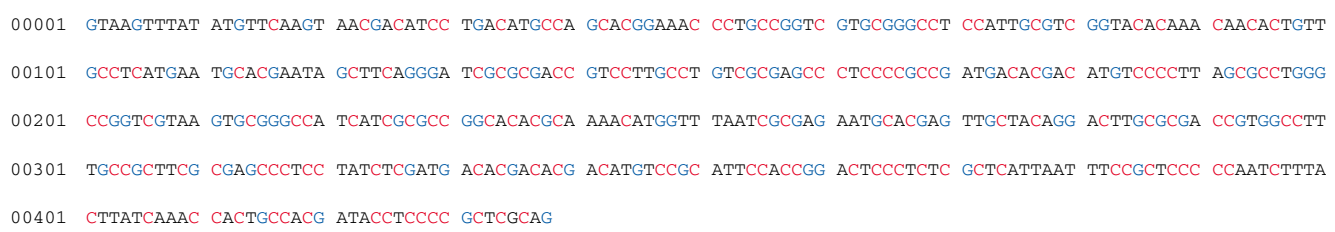

intron 2

00001 Gtgagtgagt GGTGGCGGCA AgCAAgtAgt CAgtTCGgCA GCTACTGCAA TTTCCTGCAG CTACAgCCCT GCCTGCCCGT CATGCATGCC CACCACTACC

00101 ACCGTACGCC CACCCAACCC CGCACCCCCG CGTGCCCCCC CCCCCCCCCA CACACACACA CACCCACACC TCCTTGAGGA CAGACCACCA CACTCGGCCG

00201 TGCCCCCTGC CTTGCCCACC CACCTGCAG

intron 3

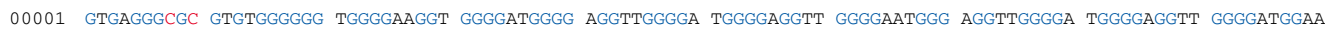

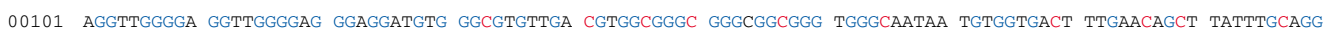

00201 AgCGCCAGCC TGCCACTGAC CTTGGCCCTC CCGCATATAC TTTCAGAgCA CAGCAGCCGT ATtGGTACTg TAgCAAGCGT GTCTGCGTGC GTGTGCCCGC

00301 CTTCCTTGCA CCCCACCTCG ACGGCTAGCC CCTAGCCCCC TCTACGTCTA GCCCCCTCCT CATCCCCTCC TTCACGCCCC GCCATTCATA CCCCTAATTG

00401 AgACCTGTTT CTGTGGGCAT GGGCGCGCGC AgGCACATAg CGCCCACCCT CCCCTCACGG CTTTCTTTGG GCTCGGGCAC ACAACACCCC ACCCCCCATC

00501 CCCCCCATCC CСTCCTGTGT CCTCCCTCCC CACAG

intron 4

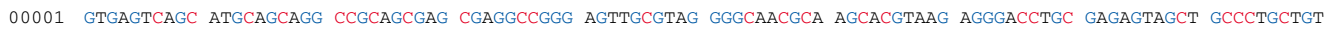

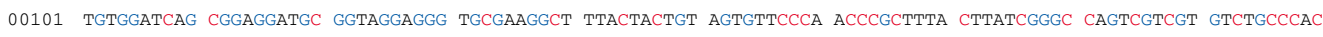

00201 GTGTGCCCAA CATTGCGCCG CCGCCTAATC CCACCGGGCC GGCCCTTGGC ACCTACCCTA GCCCGCCCGC CCGGCCGCGC CCCCCTCGCC AACCCCGTTT

00301 CCCCGGTGCA CGCACCCCTA CACCATGCCT CCCTCATCCC ATCTAACGGC TCCCCCACGC CCCCTGCTCG CCCTCGCTCC TGTCCCGCCG CCTCCTCACA

00401 ATCACAATCA CAG

intron 5

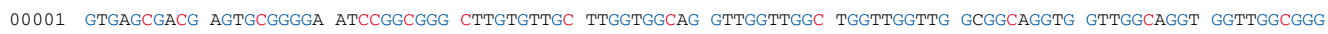

00101 TGGTTGGCGG GTGGTTGGCA GGCTGGTCGC AGGGGTTGGC ACGCATGCCA TTGTATGAGT CATCCCAATC CTCCAACCCA CCCTGCCAAT CCCCCCCAAC

00201 CCCCCAACCC ACCCCCATCC CCACCCCCCA CACACACCTA CACAG

Fig. 2. Nucleotide sequences of introns in zys3 gene. The nucleotides $\mathrm{G}$ and $\mathrm{C}$ are emphasized with blue and red, respectively. The numbers at the left of the sequences show the nucleotide position from the $5^{\prime}$ end of each intron.

CRE-BP1, CREB, and $\delta$ CREB sites at base position $780-775$ on the minus strand, and 120-kDa CRE-binding protein site at position 797-809 on the plus strand (Fig. 3A). CREB and CRE-BP1 functions as a transcription activator after modification with cAMP-dependent kinases (Yamamoto et al. 1988, Sakurai et al. 1991), while the 120-kD CRE-binding protein acts as a repressor (Andrisani and Dixon 1990). Duplication of CREs is observed upstream in mammalian genes (Inagaki et al. 1992, Someya et al. 1993, Walker et al. 1995). TESS analysis of upsteam regions of other zys gene sequences, zys $1 A$ and zys4 (zsp2-2), retrieved from database, detected two CRE-related motifs with log-likelihood scores of more than 12.0 (Fig. 3B, C). The cyclic AMP responsive element (CRE) plays an important role in mammalian spermatogenesis (Don and Stelzer 2002). Fungus mating is also regulated with cAMP (D’Souza and Heitman 2001). A Schizosaccharomyces pombe gene codes a protein similar to mammalian cAMP responsive element binding protein and disruption of this gene results in downregulation of ste11, which encodes a key transcription factor for sexual development, and deficient mating and sprorulation (Watanabe and Yamamoto 1996). Adenylyl cyclases have been isolated in several prokaryotic algae (Kasahara et al. 2001, Ohmori et al. 2002), but mating has not been reported in prokaryotic algae. Detections of cAMP in mating eu- 
A

00001 CCATGGCCCA CACCACGCGT GCCCCGCCCT GCCCCACGAC AGCTCGCGCA CACAACAATT TGCAAAGGAa AGATGGCAAC CAGAGCCGTC GATATCTGCC 00101 TTACCCTGCC TATGAGCATT GTAGCCCGTC CATAACTTTA CGGAATGCAG GGTTATCAAA CATACATAGC GCTGTGAAag TGGCGGGCAG TCCATTGCCG 00201 GCTTGCTCCC GACTGCTTGT CCCCTAGGGC GGTGGGAGTA GGGAGGGGGT CAGCGGGGAA CGAGGCTAGG AGGGAAAATT GACACCGGGT GTGCGTAGG $\overrightarrow{A P-1(12.0)}$

00301 GTTTAAGTCA CACCGCCGGC TGCAGCACCC CACATCGGGG TTGCCACTCT GCGCTCAAGT CCCGCACACG TCCCGCTCTC AGCCCATCCT TGCGCATGCI 00401 ATCAAGACAA CCGCCCAACA TTCCGGCAGC CTGACCCACA GCAGTACCCT TCCCCCCACA CATACACCCT GTCCATCCCA TTTCAGACCA GTAGCGGCCG $\overleftarrow{\mathrm{AP}-1(12.0)}$

00501 GGCGCGTATC GGTGACATGA CTGTGCCGCA CAAGCGCAAG CCATGACCGT GGCTGAGGCA CTGTGCATGC TCCTGCGCAT ATACCAGGCT TCCATTCGGA

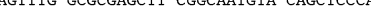

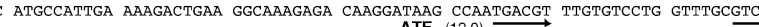
ATF-3,ATF1,ATF C-Jun,CRE-BP1,CREB, deltaCREB TREB-1 00801 CTTGCGACGg TGCAGTTTTG GGTTGCAATA CACTACTATA TAGCATACCG ATACAGCGGA GCAGAAGTTT TCGCTGAAGg GGTTGACCGT GTGTTTCTCA 00901 TGtTCGCTTT CGCTTGTCGC CACGTTtCCC CCACAATTTA CACGCGCCGA CAGGCAAACA AGATCCCCAC CAGTGCACAT CGTACGGTCA TGCACCTTGG AP-2alphâA,AP-2alphaB $(12.0)$

01001 CGTGCCCAAC CTCACAATCC CGAAGTCAGC CTGGAGCGCA TGTCATGTCA CTGACCTTGA TGCGCGACAC CGCCGGTTGG CTTTTCCGCT TGAGCCCAGC 01101 CCCCGTACCG TGTCCTCTAC GCCAACGAAT TCCAGCAGAT TTGATGCATT ACAATGACAA TACCGTGAGC TGCTGGGGGC GTGACTGGGT TCCACGACA 01201 AGCCAGCTCG CTGCTTGGCT CGCTGCCCAG CGCTGCGGCT GGTGCGCCGA AACACAGGCC GTGTCGGGCT GTGAGGGCTG TGATGTGCAG AACTCATGTC ATGATACT GITTATGCCI CGAACGATGC ATTTAGAAG AGCGCG 01401 CGCCCGGCCC GCATGGGGCA TGCCCGGCGC CCTATAAAAC GCGAGCGGAG TGCCGAACCC TGCATTCACA CAGACCT

B

00001 CTGCAGCGCC GGAACCGCAC CCGGCCTCAC CTGCTTCCAC CTGCCATCCC GAACACTCAC CCCTGCCTCC TCATACTCGG CCACACCTGC GACATGTTTC 00101 TGGCTACCTG CATCGCCGCC GGCCGCCGGC CCGCCCCTTG TCAATCGCCC CAATCACCCC AATCACCCAA ATCCCCAATC CCCCAACCCC CAATCCCCC

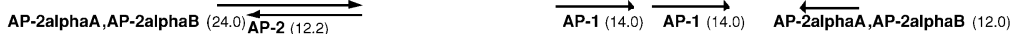
00201 CCTCCCCCCA AGTTCTATGA GGAGCTGGCG GCCCAGGGGC TGTCGCTGGC CGAGGTGTGG GAGACCGGAG CGCCCAGCCC GCCGCCCAGC CCGCCGCCGC AP-2alphaA,AP-2alphaB $(14.0)$ AP-2alphaA,AP-2alphaB $(20.0)$ -2alphaA,AP-2alphaB $(20.0)$ 00301 TCACGGAGCT GTTCCCCGAT ATGCGGGTGG CGGTGTTCCA GATAGGGCAG CAGCCGCCGC CAGCTGATGA GGAGAGGAGC TGAGTGAGGG GGGGGGCTTG 00401 GGGCTTGGAG CGGGTTCGAG CGGGTTGGA AGGGGTTTGG TGGGTTTGAT GGGTTTAGAG GGGCGGGCGT GGTTCTGGTG TTGGGTTGAG GAGGTGCGCT 00501 GTAGGCGGGG CGGGGCATGA CGGTGCTGA TGTGTGTGGG GGGGGAAGG TTTTCGTGGA CTTGACGTGA CCCGTGTGAA GGGCGAGGA AAGGCGGCG ATF-3,ATF1,ATF, C JUn, CRE-BP1

00601 GCGTCCGTGC GCGGTGTTTT TGATGGTTGG ATGGACTGAA GGACAAGTCA GGGCGAGTGG AGTGTTACAA TCAGGTTTGC ATTCCCTTGG TATATCAGGC 00701 AATTTTGAGG GGAATCAGTT ATTGTGCGTT AGGCAACTTC GCACGGCGCC AGTAACCAGT GGGGGCCACG TGACGGGTGG GATTGGGAAG GCGCATCGG 00801 CCTGCCGCGC CGCACCGCAT AAATGCGTGA AGCCGGCACC GACAAGCC

00001 GGGGGCACAG GAACGGACGG CGGGTGGGTG TGCAGGCACG GGGTCCAGGG GCGGGCTCAG GGGCGTGCGC ACTGTGCGGG GATGCAGCGC GCGATGGACC 00101 ACGCAAACTA CTACGGTATG GACTCAGCAG CCCTCGTCCA TCCTCATCCG TGTCAATCCT TGCTATGTGC CCCCTGGCGC CCACAGGTGC TGCAGACAGC 00201 GCTCTTCACG CCGCGTGTGG GCCGCCTGGG CGGGCTGTCC CCGCGCCACG AGCTCGGCGG CGGCGGCGGC GGCGGCTTCC CGCCGCACCC GCTGCGGTCG $\overrightarrow{\mathrm{AP}-2(14.0)}$

00301 GCCGCCTCGG ACAGCCGCCC GCTIGACCCG CTGTCGAGCG GCCCCTTGCC CTCCGCAGAC CCACTGTCGA CGCCGCCGCT GCTGTACGGC GCCGGTGGCA AP-1 (12.0)

00401 GCGGCGACGT GGCGGCGCC GGGTCCAACC TGGAGCTGCG GCGGGTGGGC GGCGCGGCCA CGACAGGAGG AGCAGTAGCG GGCGGCGGCG GGGCAGGAGC AP-2alphaA,AP-2alphaB $(24.0$ 00501 AGAGGCCGGC GGTGCAGGTG GCGGTGGGGC TGGGGCTGGG GATGCGTTGT CGCCGGCGGC GAGCAGCACG AAGGGCGGG CGGTGGGCGC ATTGCTTGGT

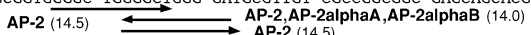
AP-2 (14.5) AP-2alphaA,AP-2alphaB $(12.0)$

00601 CGCATTCGCG CCGGCAGCGG CAAGCTGCCG GCGGCTGACG CGGAGAGCAA GGCGCCGCTG CTGGCCGGCA CAAACACGTC GCCCAGTGAC GCGGCGTGAG $\operatorname{ATF}(22.0)$

00701 TTTGCACTTC TGTCGCGGA CGGCGTGAGC GTGCGGGGCG GTCTTGCTGC GGTTGGGCCG CCATCCAGCA GTCGGTGGTG GGGGGTCGGA AAAAGGGGAG 00801 GTTGGGGCCA TGCAGCTTGG TGGAGTGGTG GTTGAGATGG AGCAGGGGAG CGCGGCAGGG CCCGCAGCCA GCTAGACCGG AGCACTGCGC GTGCGTGCAC AP-1, CREB $(12.0)$
AP-1, CREB $(12.0)$

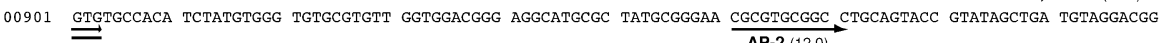

01001 GCGCCAGTGG TTGACCGGG GTTGCCGTTT TGGCGAGGCG GTCTAAAGTA GGTGTTCCCA GTCACTCAGG CTATGAGCCT TCGACGGCAG GGAGCTTCTC 01101 CTGCCCGGCG TGTGTGGATG GGGCGCTATG CAGTCACATG AATCAGGGCC GCAAGCACCA GCGTGTCCAC TACTGTCACC TGGTGTATAT GGGTTTGGAT

01201 GATTGGGTAC TTCGTGTGGC CAGGCGTGCA GAATGTTTAG GATGCGGGCG TGTGTTGCTC AGGTCGAAAC GTAAGGCTTG TATTGTATTA GCTGCTGGTG AP-1 (14.0) AP-2alphaA,AP-2alphaB (16.0)

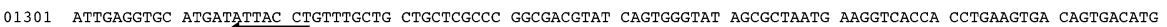
AP-1,C-Jun,Fra-1 (14.0) $\underset{\text { AP-1 (16.0) }}{\longrightarrow}$

01401 CCCCCAAACA TGGCTGGGT GTCATGTCCA AGTAGAGAAT GTCAACAAGT CGCCGTTGGA ACACACAATG GTCACTCGTG TAACATTCAA CGTGTGTAGA 01501 GGTGAGTGGC ATGGATGGCA TGGTCTTTAA TGGGATGAAC GGCGTGGGGT TGCGCCGCGG ACATGTCTCG TCGTGCTGAG CGACATGCGA CATGCACGAC 120-kDa CRE-binding protein (14.0)

01601 AACCCGTAGC TGTGGTGCCT CGCTCATAAA AGCCGTCGGT TGTGAAATAC ATT

Fig. 3. The positions and log-likelihood scores of possible binding sites of transcription factors related to AP-1 along with the nucleotide sequence of the zys3 upstream analyzed. Panel A: The 1477-bp of pC3-4 sequence upstream of 5' end of zys 3 cDNA clone, T106, is subjected to combined analysis in TESS, and AP-1 related elements detected with a log-likelihood score of more than 12.0 are shown. The numbers at the left of the sequence show the nucleotide position from the $5^{\prime}$ end (See Fig. 1) of the 5-kb region sequenced. Element and log-likelihood score are shown below underlines indicating corresponding nucleotide position on the plus $(\rightarrow)$ or minus $(\leftarrow)$ strand. Panels B and C: Upstream region sequences for zys4 (zsp2-2) and zys 1A, respectively. 
karyotic green algae, Chlamydomonas eugametos and C. reinhardtii, were reported in the 1980's (Pijst et al. 1984, Pasquale and Goodenough 1987), however, there have been no reports on CREbinding proteins in these algae. In a model higher plant, Arabidopsis, there is very little information on cAMP responsive elements. In lily pollen tubes, distributive changes of kinase-related cAMP, which occurs with the activity of pollen-tube-specific adenylyl cyclase, affects the pollen tube growth axis (Moutinho et al. 2001). In another lily, low cAMP content in pistils is related to self-incompatibility (Tsuruhara and Tezuka 2001). In mating of Chlamydomonas, flagellar protein-tyrosine kinase is related to cAMP increase (Wang and Snell 2003). Development of a genome project in $C$. reinhardtii would be a great advantage for the detection of possible CRE-related element in the future.

CRE is bound to dimers of AP-1 related factors (Watanabe and Yamamoto 1996), and functional differences are observed among different dimer components. We searched for AP-1 related elements in the zys $35^{\prime}$ upstream region. Fig. 3A shows the positions of zys 3 gene upstream putative elements of AP-1 at base position 247-252 on the plus strand and at 437-432 on the minus, ATF (Takeda et al. 1995) at 775-780 on the plus, ATF-3, ATF1, ATF, c-Jun (Hsu et al. 1992), TREB-1 at $780-775$ on the minus, AP- $2 \alpha \mathrm{A}, \mathrm{AP}-2 \alpha \mathrm{B}$ (Imagawa et al. 1987) at 969-964 on the minus. As a comparison, we have performed TESS analyses of the zys 3 introns. The results revealed detection of AP-1 motif-like sequences in intron 4 and AP-2 motif-like sequences in introns 4 and 5. Although this result points out the necessity for future analyses using binding assays of the zys 3 upstream region and element proteins, the possible zys3 upstream motifs detected suggest the existence of protein(s) that may associate with CRE-related proteins and could have a function in the mating of Chlamydomonas. Cycloheximide enhanced transcript accumulation of zys $1 B, z y s 2, z y s 3$, and zys 4 in a late mating stage, while that of other zygote-specific genes was not enhanced (Uchida et al. 1993, Ferris and Goodenough 1987). This suggests that protein factor(s) synthesized de novo may downregulate transcription of zys $1 B, z y s 2, z y s 3$, zys 4 genes in the late mating stage, or enhance degradation of the corresponding transcripts. A de novo synthesized factor(s) that might associate with CREB-like element(s) may influence individual zys gene expression early in the initiation of Chlamydomonas mating.

\section{Acknowledgements}

The authors appreciate kindness of Dr. Setsuo Koike, National Agricultural Research Center for Tohoku Region, for his support of this work, Dr. Joe Zuccarello, National Herbarium Netherlands Leiden, for his critical reading of the manuscript, Drs. Hideo Kuriyama, RIKEN, Haruko Kuroiwa, University of Tokyo, for providing revised zys3 cDNA sequences.

\section{References}

Andrisani, O. and Dixon, J. E. 1990. Identification and purification of a novel 120-kDa protein that recognizes the cAMP-responsive element. J. Biol. Chem. 265: 3212-3218.

Don, J. and Stelzer, G. 2002. The expanding family of CREB/CREM transcription factors that are involved with spermatogenesis. Mol. Cell. Endocrinol. 187: 115-124.

D’Souza, C. A. and Heitman, J. 2001. Conserved cAMP signaling cascades regulate fungal development and virulence. FEMS Microbiol. Rev. 25: 349-364.

Ferris, P. J. and Goodenough, U. W. 1987. Transcription of novel genes, including a gene linked to the mating-type locus, induced by Chlamydomonas fertilization. Mol. Cell. Biol. 7: 2360-2366.

Gilbert, S. F. 1988. Developmental Biology. Sinauer Associates, Sunderland.

Harris, E. H. 1989. The Chlamydomonas Sourcebook. Academic Press, San Diego.

Hsu, J., Bravo, R. and Taub, R. 1992. Interactions among LRF-1, JunB, c-Jun, and c-Fos define a regulatory program in the $\mathrm{G}_{1}$ phase of liver regeneration. Mol. Cell. Biol. 12: 4654-4665.

Imagawa, M., Chiu, R. and Karin, M. 1987. Transcription factor AP-2 mediates induction by two different signal-transduc- 
tion pathways: protein kinase C and cAMP. Cell 51: 251-260.

Inagaki, N., Maekawa, T., Sudo, T., Ishii, S., Seino, Y. and Imura, H. 1992. c-Jun represses the human insulin promoter activity that depends on multiple cAMP response elements. Proc. Natl. Acad. Sci. U.S.A. 89: 1045-1049.

Kasahara, M., Unno, T., Yashiro, K. and Ohmori, M. 2001. CyaG, a novel cyanobacterial adenylyl cyclase and a possible ancestor of mammalian guanylyl cyclases. J. Biol. Chem. 276: 10564-10569.

Kuriyama, H., Takano, H., Suzuki, L., Uchida, H., Kawano, S., Kuroiwa, H. and Kuroiwa, T. 1999. Characterization of Chlamydomonas reinhardtii zygote-specific cDNAs that encode novel proteins containing ankyrin repeats and WW domains. Plant Physiol. 119: 873-884.

Moutinho, A., Hussey, P. J., Trewavas, A. J. and Malhó, R. 2001. cAMP acts as a second messenger in pollen tube growth and reorientation. Proc. Natl. Acad. Sci. U.S.A. 98: 10481-10486.

Ohmori, M., Terauchi, K., Okamoto, S. and Watanabe, M. 2002. Regulation of cAMP-mediated photosignaling by a phytochrome in the cyanobacterium Anabaena cylindrica. Photochem. Photobiol. 75: 675-679.

Pan, J. and Snell, W. J. 2000. Regulated targeting of a protein kinase into an intact flagellum. J. Biol. Chem. 275: 24106-24114.

Pasquale, S. M. and Goodenough, U. W. 1987. Cyclic AMP functions as a primary sexual signal in gametes of Chlamydomonas reinhardtii. J. Cell Biol. 105: 2279-2292.

Pijst, H. L. A., van Driel, R., Janssens, P. M. W., Musgrave, A. and van den Ende, H. 1984. Cyclic AMP is involved in sexual reproduction of Chlamydomonas eugametos. FEBS Lett. 174: 132-136.

Sakurai, A., Maekawa, T., Sudo, T., Ishii, S. and Kishimoto, A. 1991. Phosphorylation of cAMP response element-binding protein, CRE-BP1, by cAMP-dependent protein kinase and protein kinase C. Biochem. Biophys. Res. Com. 181: 629-635.

Someya, Y., Inagaki, N., Maekawa, T., Seino, Y. and Ishii, S. 1993. Two 3',5'-cyclic-adenosine monophosphate response elements in the promoter region of the human gastric inhibitory polypeptide gene. FEBS Lett. 317: 67-73.

Takeda, T., Toda, T., Kominami, K., Kohnosu, A., Yanagida, M. and Jones, N. 1995. Schizosaccharomyces pombe atf ${ }^{+}$encodes a transcription factor required for sexual development and entry into stationary phase. EMBO J. 14, 6193-6208.

Tsuruhara, A. and Tezuka, T. 2001. Relationship between the self-incompatibility and cAMP level in Lilium longiflorum. Plant Cell Physiol. 42: 1234-1238.

Uchida, H., Kawano, S., Sato, N. and Kuroiwa, T. 1993. Isolation and characterization of novel genes which are expressed during the very early stage of zygote formation in Chlamydomonas reinhardtii. Curr. Genet. 24: 296-300.

—, Suzuki, L., Anai, T., Doi, K., Takano, H., Yamashita, H., Oka, T., Kawano, S., Tomizawa, K., Kawazu, T., Kuroiwa, H. and Kuroiwa T. 1999. A pair of invertedly repeated genes in Chlamydomonas reinhardtii encodes a zygote-specific protein whose expression is UV-sensitive. Curr. Genet. 36: 232-240.

- Tanifuji, G. and Hara, Y. 2001. Isolation and Characterization of a Genomic Clone of the Zygote-specific zys 3 Gene in Chlamydomonas reinhardtii. International Symposium of the Asian Pacific Phycological Association, Abstract, p. 47.

Walker, W. H., Fucci, L. and Habener, J. F. 1995. Expression of the gene encoding transcription factor cyclic adenosine 3',5'-monophosphate (cAMP) response element-binding protein (CREB): regulation by follicle-stimulating hormone-induced cAMP signaling in primary rat Sertoli cells. Endocrinology 136: 3534-3545.

Wang, Q. and Snell, W. J. 2003. Flagellar adhesion between mating type plus and mating type minus gametes activates a flagellar protein-tyrosine kinase during fertilization in Chlamydomonas. J. Biol. Chem. 278: 32936-32942.

Watanabe, Y. and Yamamoto, M. 1996. Schizosaccharomyces pombe pcrl $1^{+}$encodes a CREB/ATF protein involved in regulation of gene expression for sexual development. Mol. Cell. Biol. 16: 704-711.

Woessner, J. P. and Goodenough, U. W. 1989. Molecular characterization of a zygote wall protein: an extensin-like molecule in Chlamydomonas reinhardtii. Plant Cell 1: 901-911.

Yamamoto, K. K., Gonzalez, G. A., Biggs III, W. H. and Montminy, M. R. 1988. Phosphorylation-induced binding and transcriptional efficacy of nuclear factor CREB. Nature 334: 494-498. 